\title{
An Improved MAC Protocol with a Reconfiguration Scheme for Wireless e-Health Systems Requiring Quality of Service
}

\author{
Óscar Gama, Paulo Carvalho \\ Department of Informatics \\ University of Minho \\ Braga, Portugal \\ \{osg, pmc\}@di.uminho.pt
}

\author{
J. A. Afonso, P. M. Mendes \\ Department of Industrial Electronics \\ University of Minho \\ Guimaraes, Portugal \\ \{jose.afonso,paulo.mendes\}@dei.uminho.pt
}

\begin{abstract}
To provide a reliable assistance to patients, emergency and intensive care systems have increased demands for quality of service at different levels, including at the MAC layer. The Low Power Real Time (LPRT) MAC protocol presents suitable characteristics to address these demands due to its efficient bandwidth allocation, low energy consumption, and bounded latency. Yet, this MAC protocol may still present a significant packet loss ratio in a wireless channel affected by errors. In order to improve its robustness to bit error conditions, a solution based on short size beacons is proposed and tested. Results show that significant improvements regarding packet loss ratio and energy saving are obtained. A new reconfiguration scheme is also proposed so that a WSN using this solution may react optimally in accordance with the patients' clinical state.
\end{abstract}

Keywords: e-Health; e-Emergency; Medium Access Control; Quality of Service; Wireless Sensor Network

\section{INTRODUCTION}

At the low level, an e-health monitoring system commonly consists of a group of sensor nodes attached non-invasively to a patient in order to monitor some physiological parameters. In case of emergency clinical scenarios, a healthcare network should provide quality of service (QoS) functionalities since these scenarios clearly demand for high reliability, guaranteed bandwidth and short delays [1]. Therefore, communication protocol layers need to assure a reliable and timely data delivery, with the MAC layer assuming special relevance.

Many Medium Access Control (MAC) protocols have been developed for wireless networks using contention or multiplexing-based algorithms. Traditional contention-based protocols assume traffic is distributed stochastically. As traffic in a wireless sensor network (WSN) tends to be highly correlated and dominantly regular, conventional Carrier Sense Multiple Access (CSMA) protocols are not advised for WSNs [2]. S-MAC [3] and WiseMAC [4] are typical examples of CSMA-based protocols designed for low duty-cycle WSNs. These protocols were developed to help saving energy in applications whose nodes remain idle for long time until an event is detected (e.g. surveillance). Such protocols are convenient for WSNs having usually low traffic loads, but inadequate for networks requiring high throughput and low latency.

Amongst multiplexing-based protocols, Time Division Multiple Access (TDMA) is a commonly used technique. Time is divided into slots, which are used by motes (sensor nodes) to transmit data without the need to contend for the medium. If a base-station (BS) is available to keep the WSN scheduling and synchronization, then TDMA-based MAC protocols are usually a preferable choice to satisfy the QoS requirements of e-emergency systems, as QoS is easier assured in a collisionfree environment than in a contention-prone medium.

Within TDMA-based MAC protocols, LPRT [5] is a convenient choice to provide efficient bandwidth allocation, low energy consumption, and bounded latency, as required by e-emergency wireless networks.

LPRT is a simple, beacon-based protocol that uses dynamic and efficiently the available bandwidth. Its highly-grained superframe starts with the transmission of a beacon frame by the BS, followed by the Contention Access Period (CAP). The CAP may be used for the (dis)association or configuration of a body sensor network (BSN). The Contention Free Period (CFP) follows the CAP. The CFP is composed by the Normal Transmission Period (NTP) and the Retransmission Period (RP). NTP is used for motes to transmit new data. Lost data are retransmitted in the RP. Data packets are sent in contiguous slots of the CFP. The allocation of slots in CFP is announced in the beacon sent by the BS. Data frames transmitted during NTP are acknowledged by the ACK bitmap present in the beacon of the next superframe. LPRT uses relatively large size beacons, a single retransmission procedure, and data is only transmitted in the superframe if the corresponding beacon is received. These three characteristics may lead to a significant packet loss ratio if communications occur in a wireless channel with an appreciable bit error ratio (BER). In order to improve its robustness against bit errors, a solution based on short size beacons is proposed. Furthermore, a new algorithm to reconfigure a short size beacon $\mathrm{WSN}$ is presented. 
The remaining of this paper is organized as follows; the improvements on LPRT protocol are described in Section II; the testbed and the simulation results are presented in Section III; a new reconfiguration scheme for short size beacon WSNs is explained in Section IV; and, finally, the conclusions are discussed in Section V.

\section{IMPROVED LPRT}

To help assuring a good performance of an e-health WSN coordinated by the BS, the percentage of lost beacons should be very low. A strategy to accomplish such goal is sending beacons with a convenient transmission power, since the BER of the channel decreases as the signal-to-noise ratio increases. In addition, the beacon frame length should be as small as possible, since the probability $\mathrm{p}$ of successfully receiving a packet is:

$$
p=(1-B E R)^{8 f}
$$

where $f$ is the total length of the frame in bytes. So, the beacon payload should contain minimal essential information. The energy consumption in the WSN decreases too, since smaller size beacons are received by the motes. improved-LPRT ( iLPRT) is a MAC protocol that takes advantage of these features.

iLPRT was developed to improve some drawbacks exhibited by LPRT when it is applied in e-health systems. Since patients in emergency or intensive care situations are normally monitored by the same number and types of motes, advantage can be taken from this characteristic to decrease the packet loss ratio and energy consumption in every BSN. For this goal, iLPRT uses short size beacons, i.e. beacons carrying only the essential information for the proper operation of the WSN, which contains one or more BSNs.

Most of the time, the ACK bitmap and the CAP size of the current superframe (assuming the start slot of the CAP is known) are the only data required for the correct operation of an e-health WSN. The ACK bitmap acknowledges the new frames delivered to the BS during the last superframe. The CAP is required to allow the association of a new BSN into the WSN. In this case, since slot allocation is not sent in the beacon, motes must run an algorithm to compute which slots should be used to transmit data in the superframe without interfering with each other, in accordance with a predefined order scheme. For example, let us assume that the signals being monitored are temperature $(\mathrm{T})$, oximetry (OXI), arterial pressure (ART), respiration rate (RR) and electrocardiography (ECG), and the slots in the superframe are occupied in the following order:

beacon $\mid \mathrm{CAP}$ | RP | T(5-0),RR(5-0),OXI(5-0),ART(5-0),ECG(5-0) |

As observed, iLPRT divides the superframe in three periods: CAP, RP, and NTP. After the beacon come successively the CAP, the RP, and the NTP. ECG(5-0) = $\operatorname{ECG}(5,4,3,2,1,0)$ represents the following transmission sequence in the NTP: after ECG mote of BSN 5 (ECG5) transmitting its packet, then ECG4, ECG3, ECG2, ECG1, and ECG0 transmit successively their data. The same criterion is applied to the remaining types of motes. This regularity permits each mote to calculate its slot allocation by itself.

Each mote must calculate also the slots to retransmit its data in RP. The retransmission order depends on the ACK bitmap received from the BS. For example, using an increasing slot sequence, firstly data of all motes having the highestpriority and the bit false in the ACK bitmap are retransmitted successively. Then, data of all motes having the second higherpriority and the bit false in the ACK bitmap are retransmitted successively, and so on. If slots are not enough to permit all required retransmissions, then schedule truncation is done in order to guarantee that no retransmission occurs in RP.

With this distributed approach, if a mote does not receive a beacon or a short sequence of beacons, it may continue to send its new data in the NTP, since a mote clock drift in the order of microseconds should permit the WSN to continue synchronized during a few consecutive beacon intervals. However, in this case the mote cannot retransmit data in the RP of the superframe. As the ACK bitmap is not available, the mote cannot compute the slot allocation in the RP, neither if the data transmitted in last NTP was lost. A solution to minimize this problem is to retransmit in the CAP the data sent during the last NTP, using the slotted CSMA/CA algorithm [8]. This procedure should improve the packet loss ratio at expense of some energy consumption and CAP slots waste, since the mote may be transmitting a duplicated packet. Indeed, a packet already received by the BS may be retransmitted again in the CAP of the next superframe if the mote does not receive the beacon.

\section{Simulation Testbed AND Results}

To study the improvement obtained over LPRT using iLPRT, a case-study was setup in Castalia simulator [6]. Castalia is an open-source, discrete event-driven simulator designed specifically for wireless sensor networks. It uses the communication model proposed by Zuniga et. al. [7].

\section{A. Case-study}

A hospital room contains six beds with one patient per bed. Each patient is monitored through a BSN, and one BS collects and analyzes the vital signs of all patients. The signals being monitored are T, OXI, ART, RR, and ECG. Each signal is collected and transmitted by a dedicated mote at $250 \mathrm{kbps}$. ECG motes sample the physical signal at $250 \mathrm{~Hz}$, ART motes at $120 \mathrm{~Hz}, \mathrm{OXI}$ motes at $60 \mathrm{~Hz}, \mathrm{RR}$ motes at $20 \mathrm{~Hz}$, and T motes at $2 \mathrm{~Hz}$ [1]. The samples of every mote have a resolution of 16 bits. Knowing that the network must have a maximum latency of $500 \mathrm{~ms}$ [1], a beacon interval of $220 \mathrm{~ms}$ and 512 slots per superframe were chosen, which means that the duration of each slot is around $0.43 \mathrm{~ms}$. Consequently, packets transmitted from ECG, ART, OXI, RR, and T motes present a payload of $110 \mathrm{~B}, 54 \mathrm{~B}, 28 \mathrm{~B}, 10 \mathrm{~B}$, and $2 \mathrm{~B}$, respectively.

Since many commercial motes use ZigBee, a physical layer frame having a total maximum size of 133 bytes (B) was assumed, the same length as specified in IEEE 802.15.4 [8]. Its 
physical header of $6 \mathrm{~B}$ was also adopted: preamble sequence (4B), start frame delimiter (1B), and frame length (1B).

A MAC header of $6 \mathrm{~B}$ was tailored to this case-study, containing the fields: frame control (1B), sequence number (1B), destination address (1B), source address (1B), WSN identification (1B), and frame check sequence (2B), as shown in Figure 1. The frame type subfield identifies if it is a beacon, a data, an ACK, or a MAC command frame. The reconfiguration in progress, and the beacon received subfields are explained in the next section.

\begin{tabular}{|l|c|c|c|c|c|c|}
\hline OCTETS: 1 & 1 & 1 & 1 & 1 & VARIABLE & 2 \\
\hline $\begin{array}{l}\text { FRAME } \\
\text { CONTROL }\end{array}$ & $\begin{array}{l}\text { SEQUENCE } \\
\text { NUMBER }\end{array}$ & $\begin{array}{l}\text { DESTIN } \\
\text { ADDRESS }\end{array}$ & $\begin{array}{l}\text { SOURCE } \\
\text { ADDRESS }\end{array}$ & WSN ID & PAYLOAD & FCS \\
\hline & \\
\hline BITS: $0-1$ & 2 & 3 & $4-5$ & $6-7$ \\
\hline $\begin{array}{l}\text { FRAME } \\
\text { TYPE }\end{array}$ & ACK & $\begin{array}{l}\text { RECONFIGU } \\
\text { REQUESTION IN }\end{array}$ & $\begin{array}{l}\text { BEACON } \\
\text { PROGRESS }\end{array}$ & RECVD & RESERVED \\
\hline
\end{tabular}

Figure 1. MAC frame format and frame control field format

\section{B. Simulation Results}

For each test run, a simulation time of one hour was defined. Extending this time would not affect significantly the results. During that time period, the BS sends around 16363 beacons, carrying in the payload:

case 1) the ACK bitmap and the complete allocation information of the CFP;

case 2) the ACK bitmap and the CAP size.

In case 1 , LPRT is basically implemented with a beacon payload length of 57B: ( 1 bit in ACK bitmap +5 bits for mote identification +9 bits for slot allocation)*30 motes. For simplicity, the retransmission slots are implicitly derived from the ACK bitmap. This is not in accordance with LPRT, as the allocation in the RP must be also specified in the beacon payload. In case 2, iLPRT is implemented with a beacon payload of 5B: ( 1 bit in ACK bitmap)*30 motes +9 bits for CAP size. No retransmissions occur in the CAP. It is assumed that BER is equal in both communication directions. It should be noticed that in case 1 a mote does not transmit data in a superframe if the respective beacon is not received, which is not true for case 2 .

The probability $\mathrm{P}$ of a fully-loaded packet to be received at destination was used in the simulation runs instead of the BER. Both parameters are related by:

$$
\mathrm{BER}=1-\mathrm{P}^{1 /\left(8^{*} \mathrm{MPS}\right)}
$$

where MPS is the maximum physical frame size. Since $\mathrm{MPS}=133 \mathrm{~B}$, the BER changes between 0 and around $6.5^{*} 10^{-4}$ as $\mathrm{P}$ decreases from 1 to 0.5 along the simulation runs. Typical values for BER in a good real wireless channel are of the order $10^{-5}$ and in a bad channel may be less than $10^{-3}$.
Figures 2 and 3 clearly illustrate the improvement achieved regarding Packet Loss Ratio (PLR=100-Packet Delivery Ratio) when short size beacons are deployed in LPRT.

Considering that each sampling performed by a sensing device consumes $0.01 \mathrm{~mJ}$ of energy, simulations show that, for $\mathrm{P}=0.75$, ECG, ART, OXI, RR, and T motes extend the lifetime by a factor of $2.2,3.1,3.6,4.0$, and 4.2 , respectively, when iLPRT is used instead of LPRT.

These results confirm that the performance of a WSN improves significantly, in terms of packet loss ratio and energy saving, if short size beacons are used. Nevertheless, a reconfiguration scheme must be present in a WSN using short size beacons in order to allow the WSN to adapt dynamically to a new monitoring scenario, as required in e-health systems.

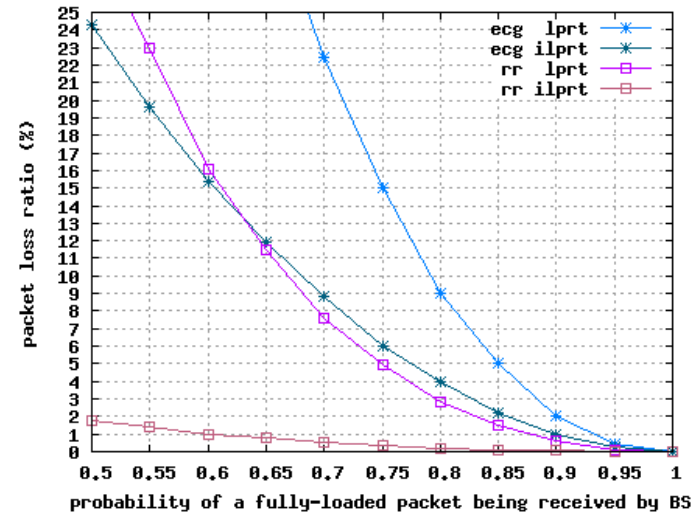

Figure 2. ECG, RR packet loss ratio for LPRT and iLPRT

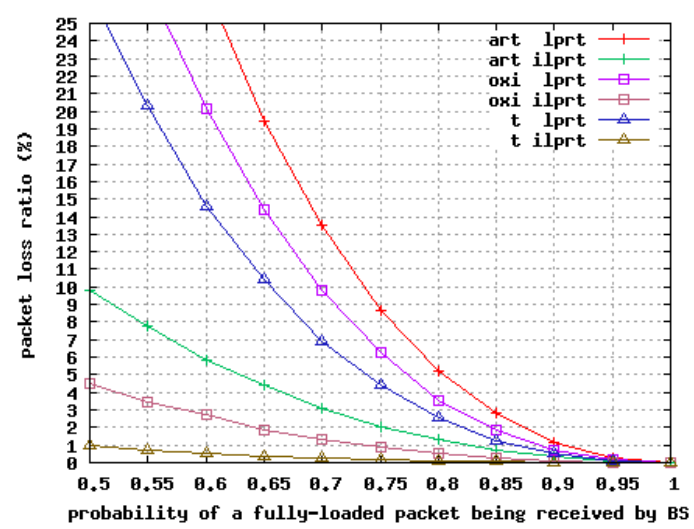

Figure 3. ART, OXI, T packet loss ratio for LPRT and iLPRT

\section{RECONFIGURATION SCHEME}

Besides the ACK bitmap and the CAP size, a beacon needs to send reconfiguration instructions if a new BSN is associated to the WSN or a new clinical situation is detected in some BSN. For instance, higher monitoring activity of the vital signs might be required when a patient's clinical situation becomes critical. 


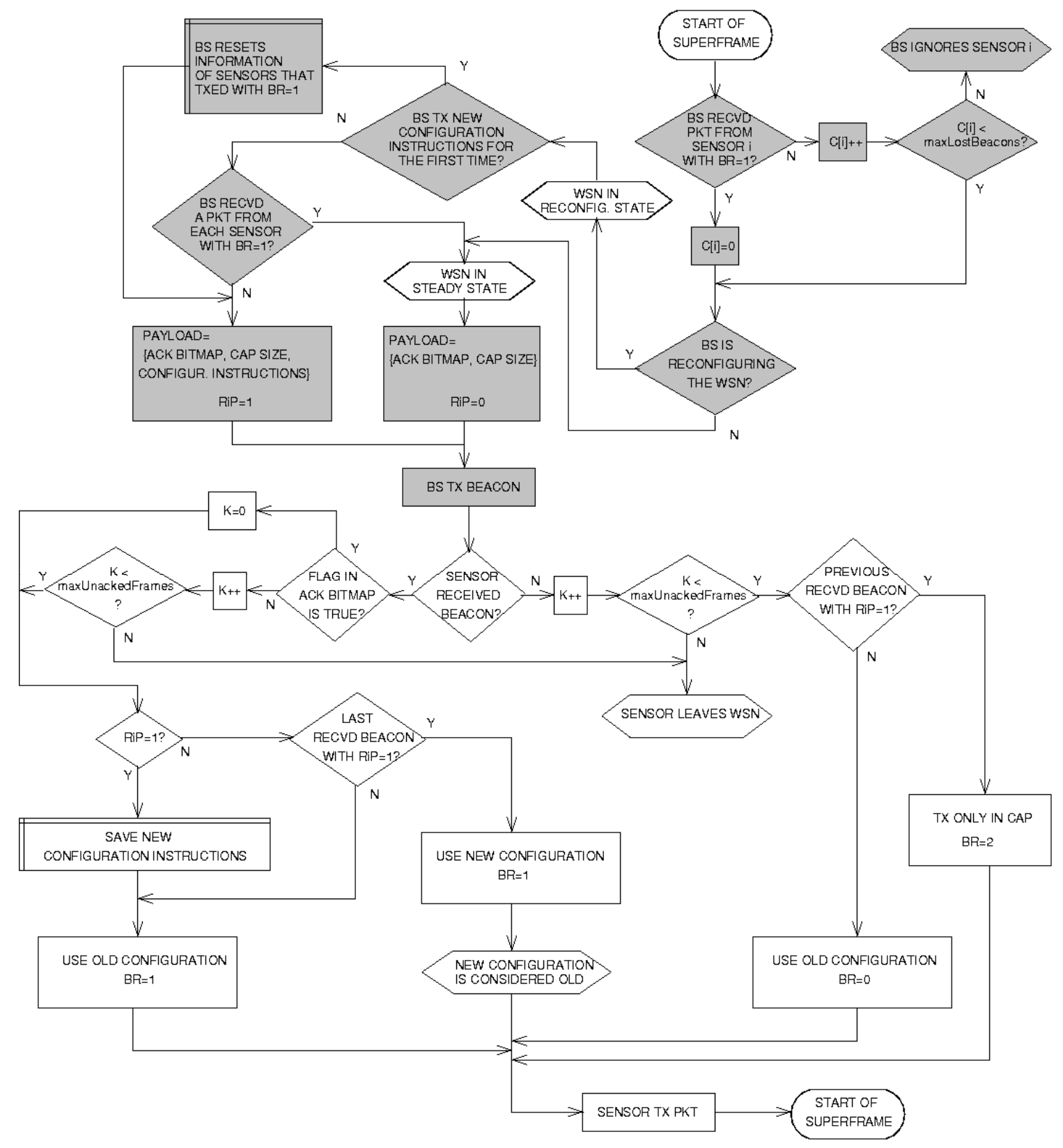

Figure 4. Reconfiguration algorithm. Grey and white blocks are relative to the BS and to the motes, respectively

In this case, the WSN should leave the steady state and enter in the reconfiguration state to adapt the network to the new situation. To perform this action in a short size beacon WSN, all motes must follow a reconfiguration scheme. Figure 4 presents the flowchart of a new algorithm specified for this purpose, which is described next.

The Reconfiguration in Progress (RiP), and the Beacon Received (BR) subfields (Figure 1) are used to reconfigure a WSN. RiP is a flag used by the BS to indicate if the WSN is in steady state $(\mathrm{RiP}=0)$ or in reconfiguration state $(\mathrm{RiP}=1) . \mathrm{BR}$ is a subfield used by the motes to acknowledge the beacons. According to Table $\mathrm{I}$, a mote sets $\mathrm{BR}=1$ whenever it receives a beacon, otherwise it makes $\mathrm{BR}=0$ or 2 , depending on if the mote ignores or not the new configuration instructions.

Let us suppose that the WSN is in the steady state. The BS sends short size beacons carrying $\mathrm{RiP}=0$, the ACK bitmap and the CAP size. Then the BS needs to reconfigure the WSN, e.g. with a new allocation of slots in the NTP. The BS sets RiP=1 and broadcasts the new configuration instructions along with 
the ACK bitmap and the CAP size. By receiving a beacon with $\mathrm{RiP}=1$, motes know that new configuration instructions are present in the beacon payload and save these instructions. However, motes continue transmitting using the old allocation of slots in the CFP. While a mote does not receive a beacon with $\mathrm{RiP}=0$, it must transmit always its packets with $\mathrm{BR}=1$ to inform the BS that the new configuration instructions have been read. If a mote does not receive a beacon and the previous received beacon contained the new configuration instructions $(\mathrm{RiP}=1)$, then it may only transmit data in the CAP with $\mathrm{BR}=2$. The identification of the motes that transmitted successfully frames with $\mathrm{BR}=1$ are registered by the $\mathrm{BS}$ in a table. If during the reconfiguration process arises another new configuration need, then the BS cleans up this table and broadcasts the recent new configuration instructions with $\mathrm{RiP}=1$. Motes must save always the instructions contained in a beacon with $\mathrm{RiP}=1$ to be informed of the last new configuration instructions. When the BS has received from each mote a packet with $\mathrm{BR}<>0$, then it knows that every mote is aware of the new configuration instructions. Hence, the BS considers the reconfiguration process complete. In the next beacon the BS sends only the ACK bitmap and CAP size, with $\mathrm{RiP}=0$. Whenever a mote receives a beacon with $\mathrm{RiP}=0$ again, it starts transmitting according to the new instructions.

If during the reconfiguration process the BS never receives a packet with $\mathrm{BR}<>0$ from a given mote, the process hangs. To prevent this, if the $\mathrm{BS}$ only received packets with $\mathrm{BR}=0$ from one mote after transmitting a number of consecutive beacons (maxLostBeacons $=16$ ), that mote becomes ignored by the BS. Also, if no data frame transmitted by a mote in the NTP is acknowledged by the respective flag in the ACK bitmap during a number of successive superframes (maxUnackedFrames $<=$ maxLostBeacons), the mote leaves the WSN at once. Both procedures are valid for the reconfiguration and steady states.

TABLE I. BR subfield of the MAC Frame Control (FC) field

\begin{tabular}{|c|c|c|c|c|}
\hline $\begin{array}{c}\text { BR } \\
\text { value }\end{array}$ & $\begin{array}{c}\text { FC } \\
\text { bits 4 5 }\end{array}$ & $\begin{array}{c}\text { Received } \\
\text { beacon? }\end{array}$ & $\begin{array}{c}\text { Mote knows the } \\
\text { new instructions? }\end{array}$ & $\begin{array}{c}\text { In which states is } \\
\text { this value used? }\end{array}$ \\
\hline 0 & 00 & no & no & steady, reconfig. \\
\hline 1 & 01 & yes & yes & steady, reconfig. \\
\hline 2 & 10 & no & yes & reconfiguration \\
\hline 3 & 11 & $\mathrm{x}$ & $\mathrm{x}$ & $\mathrm{x}$ \\
\hline
\end{tabular}

During the reconfiguration state the packet loss ratio may increase because beacons with $\mathrm{RiP}=1$ have a larger size, and also because motes do not send any data in the CFP if a beacon is not received and the last received beacon has $\mathrm{RiP}=1$. Hence, to minimize the packet loss, motes should transmit in the CAP. However, the reconfiguration state tends to occur sporadically when compared with the steady state. Hence, it is expected that the average loss ratio of a traffic flow will not be significantly affected by the inclusion of this algorithm in the network.

\section{CONCLUSIONS}

The deployment of LPRT in e-health systems leads to low power consumption, controlled latency, and throughput efficiency. However, as simulations have shown, LPRT performance is significantly affected by bit errors. In order to have a MAC protocol more robust than LPRT, the iLPRT based on short size beacons and implicit slots allocation have been proposed and evaluated. Results show that iLPRT leads to meaningful improvements regarding packet loss ratio and energy consumption. However, the short size beacon strategy is valid only if it does not compromise the reconfiguration of the e-health system. Therefore, iLPRT includes also an effective reconfiguration scheme. We believe that the strategies adopted by iLPRT are valuable to enhance the QoS provisioning in ehealth networks.

\section{ACKNOWLEDGMENT}

Óscar Gama is supported by FCT (SFRH/BD/34621/2007), Portugal.

\section{REFERENCES}

[1] Oscar Gama, Paulo Carvalho, José Afonso, P. M. Mendes, "Quality of service in wireless e-emergency: main issues and a case-study," in proc. of 3rd UCAmI, Salamanca, Spain, Oct. 2008.

[2] Jlan F. Akyildiz, Weilian Su, Yogesh Sankarasubramaniam, and Erdal Cayirci, "A survey on sensor networks," in IEEE Communications Magazine, pp.102-114, Aug. 2002.

[3] IWei Ye, John Heidemann, and Deborah Estrin, "MAC with coordinated adaptive sleeping for wireless sensor networks," IEEE/ACM Trans. Networks, vol.12, n.3, Jun. 2004.

[4] A. El-Hoiydi, J. D. Decotignie: WiseMAC, "An ultra low power MAC protocol for the WiseNET wireless sensor network," in proc. 1st ACM SenSys Conf., USA, Nov. 2003

[5] J. A. Afonso, L. A. Rocha, H. R. Silva, J. H. Correia, "MAC protocol for low-power real-time wireless sensing and actuation," 13th IEEE International Conference on Electronics, Circuits and Systems, Nice, Dec. 2006.

[6] Castalia Simulator available at:http://castalia.npc.nicta.com.au

[7] M. Zuniga, B. Krishnamachari, "Analyzing the transitional region in low power wireless links," 1st IEEE Annual Conference on Sensor and Ad Hoc Communications and Networks, Oct. 2004.

[8] IEEE Std 802.15.4-2003, Wireless Medium Access Control (MAC) and Physical Layer (PHY) Specifications for Low-Rate Wireless Personal Area Networks, Oct. 2003. 